\title{
Kernos
}

Revue internationale et pluridisciplinaire de religion grecque antique

22 | 2009

Varia

\section{Angelo Brelich, Presupposti del sacrificio umano}

\section{Pierre Bonnechere}

\section{(2) OpenEdition \\ Journals}

\section{Édition électronique}

URL : http://journals.openedition.org/kernos/1794

DOI : 10.4000/kernos. 1794

ISSN : 2034-7871

\section{Éditeur}

Centre international d'étude de la religion grecque antique

\section{Édition imprimée}

Date de publication : 1 janvier 2009

Pagination : 314-316

ISSN : 0776-3824

\section{Référence électronique}

Pierre Bonnechere, «Angelo Brelich, Presupposti del sacrificio umano », Kernos [En ligne], 22 | 2009, mis en ligne le 15 septembre 2011, consulté le 21 septembre 2020. URL : http://

journals.openedition.org/kernos/1794; DOI : https://doi.org/10.4000/kernos.1794

Ce document a été généré automatiquement le 21 septembre 2020.

Kernos 


\title{
Angelo Brelich, Presupposti del sacrificio umano
}

\author{
Pierre Bonnechere
}

\section{RÉFÉRENCE}

Angelo BRELICH, Presupposti del sacrificio umano, prefazione di Marcello Massenzio, Roma, Editori Riuniti, 2006. 1 vol. 15 臨 $21 \mathrm{~cm}, 155$ p. (Opere di Brelich). ISBN : 88-359-5847-4.

1 Ce texte réédité d'Angelo Brelich, en fait un cours d'université daté de 1967 et presque inconnu, est d'un grand intérêt. Il demeure à ce jour la plus belle tentative de présenter une vue d'ensemble du sacrifice humain de par le monde, pour en tirer des conclusions factuelles et méthodologiques. On est surpris par tant de profondeur conceptuelle, et en même temps par le refus de la conceptualisation pour elle-même. La courte préface de M. Massenzio replace l'horizon de Brelich dans une perspective historiographique, entre R. Pettazzoni et E. De Martino.

2 Si «sacrifice humain » est une juxtaposition de mots d'apparence anodine, on ne compte pas les aberrations théoriques qui furent avancées, par exemple l'idée que les Aztèques furent contraints de sacrifier des hommes faute d'animaux sacrificiels. La place réservée au sacrifice, humain et animal, varie d'une culture à l'autre, sans aucune constance, ce qui suffit à fonder l'idée que les premiers ne sont pas simplement une sous-classe abstraite des seconds, mais un réel problème historique. Brelich tente ensuite de distinguer le "sacrifice humain ", offrande faite à un être supra-humain, et les « mises à mort rituelles ", lorsque ce destinataire est absent des sources. Le concept d'«uccisioni rituali», qui seraient des rites anciens, autonomes et non cultuels, permettrait en outre de contourner d'autres difficultés méthodologiques. Dans une perspective historique, ces antécédents autonomes, les " mises à mort rituelles ", auraient été contaminés par des rites cultuels et seraient devenus, dans les systèmes polythéistes, des sacrifices humains, selon autant d'évolutions possibles qu'il y eut de sociétés supérieures. 
D'où la nécessité d'étudier ces antécédents, presupposti. En prenant garde aux contextes, Brelich se demande en quelles circonstances les mises à mort rituelles pouvaient trouver une justification culturelle aux yeux de ceux qui les accomplissent. Selon lui, le sacrifice humain n'intervient que dans une situation de crise grave, interne ou externe, crise qui ne peut être résolue par les moyens traditionnels, et qui nécessite dès lors un acte d'exception à la hauteur de sa gravité (j'ai écrit presque la même chose pour caractériser le sacrifice humain dans l'imaginaire grec, et je me réjouis de cette belle compagnie), un rachat hors du commun, qui se fonde sur une logique à mettre en lumière. La mort est antithétique : quand l'homme la subit, elle émane d'une force non humaine. Quand l'homme la donne, en ces circonstances tragiques, il agit sur le « non humain » comme un moyen de défense de l'ordre culturel : « la società che praticano le uccisioni rituali, se ne servono, per cosí dire, giocando l'anormale contro l'anormale o il sovrumano contro il sovrumano » (p. 121).

4 Ces superbes conceptions théoriques rencontrent dans la pratique bien des difficultés, et c'est tout à l'honneur de Brelich de l'avoir lui-même souligné (p. 128). Si nous possédions toute la documentation requise, on pourrait sans nul doute y accorder crédit. Mais quand on ne possède que quelques bribes d'informations, on risque de construire une continuité historique des faits qui n'est qu'illusion. C'est souvent en fonction d'un seul témoignage, évasif, tardif, rarement de visu, que nous possédons (ou non) le nom d'un destinataire suprahumain. Nous inscrivons donc le cas dans la catégorie sacrifice ou meurtre rituel sur des bases aléatoires. Il y a enfin la terminologie utilisée par chaque société pour décrire chacun des cas, et dans la société grecque, il fut autant d'explications au sacrifice humain que de tendances culturelles et sociales (et que nous aplatissons toujours en parlant «des Grecs»), mais dont de rares exemples ont subsisté : pouvons-nous tabler sur des distinctions subtiles quand nos bases sont à ce point chancelantes? Quand on dit que telle société " primitive » ne recourt pas aux mises à mort rituelles, n'est-ce pas, peut-être, parce qu'aucune trace ne nous en est parvenue ? Les mentalités évoluent, et l'on ne peut exclure non plus une évolution en dents de scie. Toute théorie axée sur une évolution progressive et inéluctable me semble indémontrable, d'autant plus que chaque société recourant au sacrifice est immédiatement confrontée, dans l'imaginaire, à l'idée que le sacrifiant puisse devenir, lui aussi, victime.

5 Le comparatisme semble permettre de combler les cases vides. Mais si chaque société a ses cases vides, ce qui est évidemment le cas, peut-on tenter d'étayer les faits au-delà des frontières culturelles, alors que la réalité interne à chaque société est déficiente ? On reste toujours prisonnier de l'angle d'étude pour chaque société, qui conditionne la signification qu'on finit par lui trouver, pour ensuite, dans la démarche comparative cette fois, travailler sur des comparables biaisés. L'ensemble des données traitées par Brelich repose sur des enquêtes de terrain et des fouilles menées entre 1880 et 1950, soit une période où le sacrifice humain était conçu comme allant de soi dans la phase " primitive » de chaque société, qui ne parvenait à s'en détacher qu'en sa période " de maturité ». Bien des rapports écrits attestent des coutumes " réelles », mais personne ne sait, en fait, - c'est le cas des Khonds en Inde par exemple, - si les récits sont dignes de confiance, ni du côté autochtone, ni du côté des colonisateurs qui fixent le récit.

6 Le problème des sources doit être repensé. Dans le $1^{\text {er }}$ chapitre, Brelich tente, avec acribie, de voir si les victimes abattues en relation avec les moments saillants d'un règne peuvent être envisagées selon une optique évolutionniste, ou encore selon un 
diffusionnisme dont il resterait alors à découvrir les canaux de diffusion. Évolutionnisme et diffusionnisme d'abord ne sont pas étanches. Ensuite, il faut compter avec l'interaction entre les traditions orales, ce que chaque peuple raconte de son passé, et la réalité, ce que chaque peuple accomplit dans les faits : nul ne sait si une pratique dite " ancestrale " n'est pas en fait une pratique récente que la tradition a couverte d'un lustre ancestral. $\mathrm{Si}$, en bien des cultures, les inhumations d'accompagnateurs mis à mort lors des funérailles semblent sporadiques dans l'espace et dans le temps, c'est peut-être parce que des pratiques mythiques furent remises « au goût du jour » avec la certitude de retourner aux usages ancestraux, puis abandonnées. Ce n'est qu'une hypothèse, mais qui met le doigt sur le simplisme de certaines reconstitutions anthropologiques. De même, si les immolations humaines lors des rites de fondation pourraient être en rapport avec la mise à mort cosmogonique d'un être dont le corps servirait de base à la nouvelle création, dans quel sens cette influence s'est-elle développée ? Peut-on vraiment supposer une strate antérieure dans la culture où une victime humaine était destinée à conforter la nouvelle création (d'une maison, d'un pont, ...), en miroir avec la création de l'Univers?

7 La $1^{\text {re }}$ partie, de loin la plus longue (p. 29-117), explore les « uccisioni rituali ». Cinq sous-chapitres sont consacrés aux funérailles royales (Mésopotamie, Égypte, préhistoire, Afrique, Orient, Chine, Amérique), aux rites de fondation, aux rites agraires (notamment au Japon), aux situations de crise et aux rites de purification, enfin au cannibalisme et à la chasse aux têtes. La $2^{\mathrm{e}}$ partie (p. 125-145) revient rapidement sur le développement de ces prémisses et leur évolution (souvent un vernis sur le rite originel) dans le cadre des polythéismes, parfois en " sacrifici umani ", parfois en aversion pour le phénomène, selon les racines de chaque société et les circonstances de leur évolution propre.

8 De façon significative, les problèmes terminologiques, qui trahissent les distinctions sémantiques qu'on tente d'y classer, demeurent irrésolus à ce jour. Lors d'une journée d'étude sur le sacrifice humain tenue à Montréal en 2008, la moitié de la discussion y fut consacrée, et un travail collectif de recherche en France, entre 1999 et 2002, connut les mêmes débats. On doit reconnaître d'ailleurs que Brelich avait magistralement ouvert la voie, même si cet écrit est resté au stade embryonnaire et si sa seconde partie demeure un plan commenté de ce qui devrait encore être fait. Enfin, en aucun cas, il ne faut se méprendre sur mes critiques : je fais ici le compte rendu d'un ouvrage de 1967, en nain juché sur ces géants qui ont développé la discipline, et dont Brelich fait assurément partie. J'aurais donné beaucoup pour assister à des cours aussi clairs, intelligents, méthodologiquement équilibrés et j'avoue être diablement jaloux des étudiants romains de cette époque.

9 Ajoutons quelques titres au complément bibliographique assez sommaire de la p. 155: Archiv für Religionsgeschichte, 1, 1999 (volume consacré au sacrifice humain); J.-P. Albert \& B. Midant-Reynes(éds), Le sacrifice humain en Égypte ancienne et ailleurs, Paris, 2005; M. Graulich, Le sacrifice humain chez les Aztèques, Paris, 2005; J.N. Bremmer (éd.), The Strange World of Human Sacrifice, Leuven, 2007; Human Sacrifice among Jews and Christians, Leiden, 2008 (Numen, suppl.112). On me permettra aussi de mentionner mon livre, paru à Liège en 1994 : Le sacrifice humain en Grèce ancienne (Kernos, suppl. 3). Une rapide enquête à " human sacrifice » dans le moteur de recherche d'une grande bibliothèque universitaire montrera l'étendue d'un domaine aussi riche que disparate sur le sujet, et souvent mal informé des avancées (et reculs) enregistrés. 


\section{AUTEURS}

\section{PIERRE BONNECHERE}

Université de Montréal 